\title{
Satisfacción con la vida, autoestima y optimismo financiero en estudiantes interculturales de México
}

\author{
Marco A. Núñez-Ramírez ${ }^{1 *}$, Rosalva I. Castro Álvarez ${ }^{2}$, Altayra G. Ozuna-Beltrán ${ }^{3}$, y \\ Rosario del C. Realpozo-Reyes ${ }^{4}$ \\ (1) Instituto Tecnológico de Sonora, Departamento de Ciencias Administrativas, 5 de febrero 818 Sur, Ciudad Obregón, \\ Sonora, México (correo-e: marco.nunez@itson.edu.mx). \\ (2) Universidad LaSalle Noroeste, Departamento de Ciencias Administrativas, Calle Veracruz s/n, Norte, Ciudad \\ Obregón, Sonora, México (correo-e: rosalva.castro.m@lasallenoroeste.edu.mx). \\ (3) Instituto Tecnológico de Sonora, Departamento de Contaduría y Finanzas, 5 de febrero 818 Sur, Ciudad Obregón, \\ Sonora, México (correo-e: altayra.ozuna@itson.edu.mx). \\ (4) Universidad Intercultural del Estado de Tabasco, Poblado de Oxolotán, Tacotalpa, Tabasco, México \\ (correo-e: realpozor@outlook.com).
}

* Autor a quien debe ser dirigida la correspondencia.

Recibido Mar. 31, 2021; Aceptado Jun. 5, 2021; Versión final Jun. 23, 2021, Publicado Oct. 2021

\begin{abstract}
Resumen
El objetivo de la presente investigación es medir la influencia de la satisfacción de la vida y autoestima sobre el optimismo financiero en estudiantes universitarios interculturales. El modelo de la universidad intercultural en México ha tratado de empoderar y desarrollar diferentes habilidades en los estudiantes, en especial entre los miembros de los pueblos originarios, quienes han sufrido problemas sociales y económicos. Mediante una muestra conformada por 455 estudiantes de universidades interculturales del norte y sur de México, se encontró que el optimismo financiero es influenciado por la autoestima y la satisfacción con la vida. Se concluye que disciplinas como la psicología positiva y la economía conductual podrían ser empleadas para explicar el rezago que experimentan los miembros de los pueblos originarios $y$, al mismo tiempo, ser consideradas en el desarrollo de programas educativos dentro del modelo de universidad intercultural.
\end{abstract}

Palabras clave: satisfacción con la vida; autoestima; optimismo financiero; universidad intercultural

\section{Satisfaction with life, self-esteem, and financial optimism in intercultural students from Mexico}

\begin{abstract}
The main objective of this research study is to measure the influence of life satisfaction and self-esteem on financial optimism in intercultural university students. The model of intercultural university in Mexico has attempted to empower and develop different skills in students, especially among members of indigenous peoples, who have suffered social and economic issues. The sample consists of 455 students from intercultural universities located in the north and south of Mexico. The results show that financial optimism is influenced by self-esteem and life satisfaction. It is concluded that disciplines such as positive psychology and behavioral economics can be applied to explain the backwardness experienced by indigenous group members and, at the same time, be considered in the development of educational programs within the intercultural university model.
\end{abstract}

Keywords: satisfaction with life; self-esteem; financial optimism; intercultural university 


\section{INTRODUCCIÓN}

En Latinoamérica, los grupos indígenas se han visto afectados por serios problemas de pobreza y desigualdad (García et al., 2021). Según Miranda (2021), en esta región la tasa de pobreza de la población indígena ha tenido un aumento durante las últimas dos décadas. En esta misma sintonía, el Instituto Nacional de los Pueblos Indígenas (2018) afirma que el $71.9 \%$ de los pueblos originarios vive en condiciones de pobreza. Además, en el caso de México, el Consejo Nacional de Evaluación de la Política Social (2020) señala que tres de cada 10 indígenas se enfrentan a rezagos en cuanto a lo educativo. Dentro del contexto latinoamericano, ha surgido evidencia empírica que ha tratado de caracterizar a la educación intercultural en la región (Villalta, 2016), a través de la cual se destaca que, en un mundo globalizado y multicultural como el presente, resulta imperante la necesidad de promover una educación que favorezca la inclusión y desarrollo personal (Johnson, 2015; Martínez et al., 2020), aspectos que, además de estar estrechamente relacionados, constituyen la base de la interculturalidad.

Aunque mayormente la interculturalidad se refiere a la interacción entre miembros de diferentes grupos culturales, a grandes rasgos, su concepción depende de la parte contextual (Johnson, 2015). Bajo este enfoque, y considerando que México es un país megadiverso y multicultural donde conviven 68 grupos etnolingüísticos con 264 variantes (Instituto Nacional de Lenguas, 2018), ha surgido el modelo de universidad intercultural, cuyos propósitos han sido el fortalecer y empoderar a los estudiantes indígenas para contribuir de manera significativa en sus comunidades a través del fomento de la cultura, identidad y la lengua (Dietz y Mateos, 2020). Éste, asimismo, procura "repensar y reformular la combinación de las tareas sustantivas de cualquier universidad -docencia, investigación y vinculación- para que las comunidades y regiones anfitrionas se puedan beneficiar directamente de la presencia de una IES" (Mateos y Dietz, 2016).

Es importante señalar que, dentro de los objetivos de este modelo de universidad, se destaca su intención por la erradicación de la pobreza y el fortalecimiento del capital humano (Tapia, 2016). En este tenor, uno de los desafíos más importantes que se ha asumido es el empoderamiento de sus estudiantes (Dietz y Mateos, 2020; Martí y Dietz, 2014), quienes en su mayoría provienen de contextos de marginación y desigualdad (García et al., 2021; Miranda, 2021). Una perspectiva emergente que podría apoyar a contrarrestar la pobreza es la educación financiera (Loomis, 2018); la cual puede ser empleada por dicho modelo con el fin de cumplir sus objetivos educativos y fomentar el desarrollo, a través de una pedagogía que integre tanto a la economía conductual como la psicología positiva.

Los grupos originarios poseen elementos culturales únicos, tales como su cosmovisión y conocimiento ancestral, aspectos que hacen la diferencia dentro de la toma de decisiones económicas y, además, son factores clave para entender las actitudes y el comportamiento financiero de tales grupos. Considerando estos aspectos, es imperante la necesidad de estudiar el efecto que tiene la educación financiera en los grupos originarios; por ello, es significativo conocer el comportamiento financiero, el cual, según Blue (2019), ayudaría a entender a los grupos en situación de vulnerabilidad. En ese sentido, la economía conductual puede favorecer la explicación de la parte actitudinal de los individuos. De hecho, esta disciplina ha favorecido a la comprensión del comportamiento de los grupos interculturales, abarcando temas como las actitudes, las percepciones, el consumo, precios, demanda, riesgos y mercados, y, además, ha identificado cómo piensan y sienten las personas (Fonseca et al., 2019). De esta perspectiva teórica se desprende el optimismo financiero, variable que puede jugar un rol relevante dentro de los propósitos que persigue el modelo de la universidad intercultural.

Según Zabelina et al. (2018), uno de los aspectos estudiados por la economía conductual, y que puede favorecer a la comprensión del comportamiento, es el optimismo financiero, el cual se define como un conjunto de actitudes que forman en los individuos la convicción de que habrá prosperidad económica. Para Talwar et al. (2021), ésta es una inclinación psicológica que se manifiesta cuando las personas son positivas y esperan mejores resultados financieros en situaciones inciertas; además, es una variable individual que refleja el grado en que las personas tienen expectativas favorables y generalizadas sobre su futuro (Dawson, 2017); y explica cómo son administradas las finanzas personales (White et al., 2021). En cuanto a los grupos interculturales, aunque existe evidencia empírica que sostiene que la economía conductual ha ignorado las diferencias culturales en la toma de decisiones económico-financieras (Levinson y Peng, 2007), aún no queda del todo claro cómo se caracteriza el comportamiento financiero de estos grupos; por ello es importante seguir estudiando el trasfondo cultural en la toma de decisiones económicas.

Retomando el interés por empoderar a estudiantes de grupos vulnerables por parte del modelo de la universidad intercultural (Dietz y Mateos, 2020; Martí y Dietz, 2014), una perspectiva que podría apoyar con este propósito dentro de la educación financiera es la psicología positiva, la cual busca promover el desarrollo de las fortalezas del ser humano (Seligman y Pawelski, 2003). Es así que, tanto la economía conductual como la psicología positiva podrían fungir como herramientas para la formación de los estudiantes dentro de contextos interculturales. 


\section{OTROS ANTECEDENTES}

Dentro de la psicología positiva, dos variables que abordan el desarrollo de las fortalezas personales son la satisfacción con la vida y la autoestima. La primera, es entendida como un juicio cognitivo, donde un individuo evalúa cuán satisfecho se encuentra acerca de su vida en comparación con sus propias expectativas (Diener et al., 1985); además, es el grado positivo de autoevaluación de la vida como un todo (Veenhoven, 2015). Según APA (2010), esta variable es "la medida en que una persona encuentra que su vida es rica, significativa, plena o de alta calidad". En segundo lugar, se encuentra la autoestima, que, en términos generales, es concebida como un sentimiento o valoración que tiene una persona sobre sí misma, de acuerdo a Rosenberg (1965), quien es uno de los pioneros en la investigación de este tema y uno de sus principales exponentes. Esta variable es, del mismo modo, "el grado de perfección de las cualidades y características personales contenidas en el propio autoconcepto como positivas" (APA, 2010). Respecto a la relación entre la autoestima y la satisfacción con la vida, ha surgido evidencia empírica que la explica. Dentro del entorno Latinoamericano, Núñez et al. (2020) encontraron una asociación significativa y positiva en estudiantes de México y Bolivia; sin embargo, a pesar de este tipo de estudios, existe poca investigación enfocada hacía un contexto intercultural, específicamente orientado a estudiantes de pueblos originarios. Esto se hace más pertinente al considerar que la cultura tiene una relación importante respecto a la interpretación de la satisfacción con la vida (Krys et al., 2021) y la autoestima (Salzman, 2018).

Es así que la educación financiera podría favorecer al modelo de universidad intercultural en países multiculturales, como es el caso de México. Esto puede comprenderse al considerar que la psicología positiva se centra en el desarrollo integral de las personas (Seligman y Pawelski, 2003), donde la parte económica también debe ser tomada en cuenta. Primeramente, es importante tomar en cuenta que la satisfacción con la vida tiene una relación significativa con desarrollo económico (Veenhoven, 2015). Es así que esta variable converge con el optimismo financiero en lo siguiente: a) ambas versan sobre expectativas futuras donde también se involucra la parte económica; b) el optimismo financiero se asocia con el bienestar económico (White et al., 2020), mientras que la satisfacción con la vida se ha integrado al bienestar subjetivo (Pavot y Diener, 2008). Por su parte, de la autoestima se deriva la percepción financiera. Entonces, si el deseo de éxito financiero se asocia con una baja autoestima (Ward et al., 2021); mientras que la autoestima se asocia de manera positiva con el optimismo (Núñez et al., 2020); por lo tanto, la autoestima podría influir sobre el optimismo financiero. A partir de esto, ¿cuál sería el rol de variables como la satisfacción con la vida y la autoestima dentro del desarrollo del optimismo financiero?

Retomando que la educación financiera dentro del contexto intercultural es un tema poco considerado, el cual requiere mayor evidencia empírica que permita proponer nuevos caminos a seguir, en especial dentro de la universidad intercultural en México, que cuenta con pocos años de existencia (Mateos y Dietz, 2016), y que tiene dentro de sus objetivos la misión de empoderar a sus estudiantes (Dietz y Mateos, 2020; Martí y Dietz, 2014); por lo tanto, es así que surge la presente pregunta de investigación: ¿Cómo influye la satisfacción con la vida y la autoestima sobre el optimismo financiero en estudiantes interculturales de México? Para dar respuesta a dicha interrogante, se plantean las siguientes hipótesis de estudio: $H_{1}$ ) A mayor satisfacción con la vida, mayor es la percepción del optimismo financiero en estudiantes interculturales de México; $H_{2}$ ): A mayor autoestima, mayor es la percepción del optimismo financiero en estudiantes interculturales de México. Cabe señalar que dicho estudio permitirá, en un primer momento, aportar evidencia empírica que favorezca la comprensión de la educación financiera actual dentro de la universidad intercultural en este país, y, al mismo tiempo, servir de insumo para la generación de propuestas pedagógicas donde se incluya a la psicología positiva y la economía conductual. A continuación, se muestra el diseño metodológico propuesto para corroborar empíricamente tales postulados.

\section{MATERIALES Y MÉTODOS}

Con el fin de probar las hipótesis de estudio, se realizó una investigación de tipo cuantitativa con alcance correlacional. A continuación, se muestra la caracterización de los participantes del estudio, la descripción de los instrumentos de medición empleados y, finalmente, el procedimiento que se llevó a cabo.

\section{Sujetos}

Luego de haber realizado un muestreo no probabilístico, se administró el cuestionario a una muestra conformada por 455 estudiantes de tres instituciones interculturales de educación superior de México: Universidad Intercultural del Estado de Tabasco $(n=245)$, Universidad Autónoma Intercultural de Sinaloa $(n$ $=139$ ), e Instituto Tecnológico Superior de Cajeme $(n=71)$. Se señala que esta última no pertenece propiamente al subsistema de Universidades Interculturales dentro de la Coordinación General de Educación Intercultural y Bilingüe (CGEIB) de México; sin embargo, ésta se encuentra ubicada en Vícam, el cual es uno de los pueblos yaquis del estado de Sonora. La Tabla 1 presenta la caracterización de los sujetos del estudio. 
Tabla 1. Caracterización de la muestra

\begin{tabular}{|c|c|c|}
\hline Características & $n$ & $\%$ \\
\hline \multicolumn{3}{|l|}{ Género } \\
\hline Femenino & 249 & 54.7 \\
\hline Masculino & 206 & 45.3 \\
\hline Edad & $M=21.2$ & $D E=4.73$ \\
\hline \multicolumn{3}{|l|}{ Área de estudio } \\
\hline Ciencias Económico Administrativas & 80 & 18 \\
\hline Ingenierías & 137 & 30 \\
\hline Ciencias Sociales & 36 & 8 \\
\hline Ciencias Médicas & 202 & 44 \\
\hline \multicolumn{3}{|l|}{ Pueblo originario de pertenencia } \\
\hline Yaqui & 60 & 13.1 \\
\hline Mayo & 9 & 2.0 \\
\hline Mixteco & 21 & 4.6 \\
\hline Tepehuano & 6 & 1.3 \\
\hline Chatino & 5 & 1.1 \\
\hline Tarahumara & 1 & .2 \\
\hline Tsotsil & 7 & 1.5 \\
\hline Zapoteco & 6 & 1.3 \\
\hline Nahuatl & 2 & .4 \\
\hline Chinanteco & 2 & .4 \\
\hline Ch'ol & 31 & 6.8 \\
\hline Zoque & 3 & .7 \\
\hline Chontal & 1 & .2 \\
\hline No miembro de pueblo originario & 301 & 66.2 \\
\hline
\end{tabular}

\section{Instrumentos de medición}

El cuestionario estuvo compuesto por tres instrumentos de medición. Primeramente, respecto a la medición de la satisfacción con la vida, se administró una adaptación al español del cuestionario SWLS (Satisfaction with Life Scale), el cual fue diseñado por Diener et al. (1985), y se compone por cinco ítems con escala Likert de siete puntos de respuesta: 1 (Totalmente en desacuerdo), 2 (Ligeramente en desacuerdo), 3 (En desacuerdo), 4 (Ni de acuerdo ni en desacuerdo), 5 (De acuerdo), 6 (Ligeramente de acuerdo), y 7 (Totalmente de acuerdo); misma escala empleada para la medición del optimismo financiero a través del uso de una adaptación del cuestionario elaborado por Zabelina et al. (2018), el cual consistió en dos ítems. Finalmente, para medir autoestima, se empleó una adaptación al español del cuestionario propuesto por Rosenberg (1965), conocido como RES (Rosenberg Self-esteem Scale), que se compone por 10 ítems en escala Likert 4 con los siguientes valores: 1 (Muy en desacuerdo), 2 (En desacuerdo), 3 (De acuerdo) y 4 (Muy de acuerdo). Para la medición de la validez y confiabilidad de las variables del cuestionario, se realizó el Análisis de Varianza Extraída Media (AVE) y Fiabilidad Compuesta, cuyos resultados se pueden apreciar en la Tabla 2.

Tabla 2. Validez y Confiabilidad de las variables

\begin{tabular}{|l|r|r|r|r|}
\hline \multicolumn{1}{|c|}{ Variables } & AVE & \multicolumn{1}{c|}{$\begin{array}{c}\text { Fiabilidad } \\
\text { Compuesta }\end{array}$} & \multicolumn{1}{c|}{ DE } \\
\hline Satisfacción con la vida & .506 & .854 & 5.11 & 1.163 \\
\hline Autoestima & .514 & .816 & 3.13 & .480 \\
\hline Optimismo financiero & .474 & .826 & 5.86 & 1.174 \\
\hline
\end{tabular}




\section{Procedimiento}

El desarrollo de la presente investigación corrió a cargo de profesores del Instituto Tecnológico de Sonora, la Universidad Intercultural del Estado de Tabasco y Universidad LaSalle Noroeste, todas éstas ubicadas en México. En un primer momento, respecto a la validación de contenido de los cuestionarios, se trabajó a través del proceso traducción-retraducción, y, posteriormente, se llevó a cabo una prueba piloto. Luego de haber obtenido autorización por las autoridades de las instituciones educativas, los cuestionarios fueron administrados por los investigadores y otros profesores de las universidades a los participantes, quienes, después de ser informados del objetivo, características y confidencialidad de la investigación, participaron de manera voluntaria, resaltando el carácter confidencial y anonimato. Con el propósito de poner a prueba las hipótesis de investigación, se llevaron a cabo las siguientes pruebas estadísticas: a) estadística descriptiva de las variables, en especial, mediante el empleo de las categorías de evaluación propuestas por Diener et al. (1985) y Rosenberg (1965), en cuanto al estudio de la satisfacción de la vida y autoestima, respectivamente; b) correlación y regresión múltiple para medir la influencia la satisfacción de la vida y autoestima sobre el optimismo financiero, empleando como variables control el género, universidad y grupo étnico. A continuación, se muestran los resultados obtenidos.

\section{RESULTADOS}

En un primer momento, se muestran los resultados descriptivos de las variables de estudio. Respecto a la evaluación de la satisfacción con la vida, a partir de la lectura de Pavot y Diener (2008), los resultados, según la puntuación obtenida, pueden ser categorizados en siete niveles: 1) extremadamente insatisfecho (5-9); 2) insatisfecho (10-14); 3) ligeramente insatisfecho (15-19); 4) neutral (20); 5) ligeramente satisfecho (21-25); 6) satisfecho (26-30); 7) extremadamente satisfecho (31-35). Las frecuencias de esta variable pueden ser observadas en la Tabla 3, donde es posible verificar que el 83.1 de los participantes mostraron niveles positivos de satisfacción $(n=378)$; mientras que, en el caso de la autoestima, mediante la categorización del RES, $91.2 \%$ mostró niveles favorables: medio y alto (Ver tabla 4).

Tabla 3: Nivel de satisfacción de la vida

\begin{tabular}{|l|l|r|r|}
\hline Valor & \multicolumn{1}{|c|}{ Nivel } & \multicolumn{1}{c|}{$n$} & \multicolumn{1}{c|}{$\%$} \\
\hline 1 & Extremadamente insatisfecho & 10 & 2.2 \\
\hline 2 & Insatisfecho & 16 & 3.5 \\
\hline 3 & Ligeramente insatisfecho & 37 & 8.1 \\
\hline 4 & Neutral & 14 & 3.1 \\
\hline 5 & Ligeramente Satisfecho & 116 & 25.5 \\
\hline 6 & Satisfecho & 170 & 37.4 \\
\hline 7 & Extremadamente Satisfecho & 92 & 20.2 \\
\hline
\end{tabular}

Tabla 4: Nivel de autoestima

\begin{tabular}{|l|r|r|}
\hline \multicolumn{1}{|c|}{ Nivel } & $n$ & $\%$ \\
\hline Bajo & 40 & 8.8 \\
\hline Medio & 117 & 25.7 \\
\hline Alto & 298 & 65.5 \\
\hline
\end{tabular}

Por otro lado, desde la parte inferencial, se pusieron a prueba las hipótesis de estudio mediante diferentes pruebas estadísticas. Primeramente, en la Tabla 5, se muestran las correlaciones donde es posible observar que las tres variables de estudio se asocian de manera significativa $(p<.001)$. Esto refleja la pertinencia para continuar con el siguiente análisis.

Tabla 5: Correlación de las variables

\begin{tabular}{|l|r|r|r|}
\hline \multicolumn{1}{|c|}{ Variables } & \multicolumn{1}{c|}{1} & \multicolumn{1}{c|}{2} & \multicolumn{1}{c|}{3} \\
\hline 1. Satisfacción con la vida & 1 & & \\
\hline 2. Autoestima & $.282^{* *}$ & 1 & \\
\hline 3. Optimismo financiero & $.435^{* *}$ & $.239^{* *}$ & 1 \\
\hline
\end{tabular}


Luego de los resultados anteriores, se pasó a la realización de regresión múltiple. Aquí, a través del empleo del estadístico Durbin-Watson y el Factor de Inflación de la Varianza (VIF), se pudo constar que los modelos no presentaron problemas en cuanto a la autocorrelación de los errores ni multicolinealidad, respectivamente. En la Tabla 6, se muestra cómo los tres modelos propuestos tienen algún impacto sobre el optimismo financiero: variables control (1), satisfacción con la vida (2) y autoestima (3). Es importante resaltar que todos los modelos propuestos, incluyendo el que considera las variables control, muestran valores significativos $(p$ $<0.01$ ), donde se resalta el nivel de explicación a la influencia de la satisfacción con la vida (23.1\%) y la autoestima $(24.8 \%)$, respectivamente.

Tabla 6: Resumen de los modelos de regresión

\begin{tabular}{|c|c|c|c|c|c|c|c|}
\hline \multirow[t]{2}{*}{ Modelos } & \multirow[t]{2}{*}{$\mathrm{R}^{2}$} & \multirow{2}{*}{$\begin{array}{c}\mathrm{R}^{2} \\
\text { ajustado }\end{array}$} & \multirow{2}{*}{$\begin{array}{l}\text { Error } \\
\text { Estándar }\end{array}$} & \multicolumn{3}{|c|}{ Estadísticas de cambios } & \multirow[b]{2}{*}{$\begin{array}{l}\text { Durbin- } \\
\text { Watson }\end{array}$} \\
\hline & & & & $\begin{array}{c}\text { Cambio de } \\
\text { cuadrado de R }\end{array}$ & $\begin{array}{l}\text { Cambio } \\
\text { en F }\end{array}$ & $p$ & \\
\hline $\begin{array}{l}1 \text { Predictores: (Constante), Género, } \\
\text { Universidad, Grupo étnico }\end{array}$ & .071 & .064 & 1.1357 & .071 & 11.332 & .000 & \multirow{3}{*}{1.846} \\
\hline $\begin{array}{l}2 \text { Predictores: (Constante), Género, } \\
\text { Universidad, Grupo étnico, } \\
\text { Satisfacción con la Vida }\end{array}$ & .231 & .224 & 1.0341 & .161 & 93.326 & .000 & \\
\hline $\begin{array}{l}3 \text { Predictores: (Constante), Género, } \\
\text { Universidad, Grupo étnico, } \\
\text { Satisfacción con la Vida, Autoestima }\end{array}$ & .248 & .240 & 1.0235 & .017 & 10.295 & .001 & \\
\hline
\end{tabular}

Retomando $\mathrm{H}_{1}$, la cual sostiene que, a mayor satisfacción con la vida, mayor es la percepción del optimismo financiero en estudiantes interculturales de México, los hallazgos reflejaron que la variable independiente influye positivamente sobre el optimismo financiero $(\beta=.405 ; t=9.661 ; p<.001)$, lo cual soporta empíricamente dicha postulación. Además, es importante señalar que ciertas variables control como la universidad y la pertenencia a algún grupo étnico también obtuvieron resultados significativos. Esto puede ser interpretado a la luz de un enfoque cultural. Por otro lado, los hallazgos también prueban la segunda hipótesis, debido a que, en la Tabla 7, puede observarse que la autoestima también presenta un impacto positivo sobre la variable dependiente $(\beta=.138 ; t=3.209 ; p \leq .001)$. Se resalta que los efectos de las variables independientes se producen aun cuando se emplearon variables control dentro de los modelos.

Tabla 7: Coeficientes de los modelos de regresión

\begin{tabular}{|c|c|c|c|c|c|c|c|c|}
\hline & & \multicolumn{2}{|c|}{$\begin{array}{l}\text { Coeficientes no } \\
\text { estandarizados }\end{array}$} & \multirow{2}{*}{$\begin{array}{c}\begin{array}{c}\text { Coeficientes } \\
\text { estandarizados }\end{array} \\
\text { Beta }\end{array}$} & \multirow[b]{2}{*}{$t$} & \multirow[b]{2}{*}{$p$} & \multicolumn{2}{|c|}{$\begin{array}{l}\text { Estadísticas de } \\
\text { colinealidad }\end{array}$} \\
\hline \multicolumn{2}{|c|}{ Modelos } & B & $\begin{array}{c}\text { Error } \\
\text { estándar }\end{array}$ & & & & Tolerancia & VIF \\
\hline \multirow[t]{4}{*}{1} & (Constante) & 6.675 & .194 & & 34.385 & .000 & & \\
\hline & Género & -.055 & .109 & -.024 & -.508 & .612 & .966 & 1.035 \\
\hline & Universidad & -.390 & .073 & -.246 & -5.326 & .000 & .969 & 1.032 \\
\hline & Grupo étnico & -.039 & .017 & -.109 & -2.361 & .019 & .978 & 1.023 \\
\hline \multirow[t]{5}{*}{2} & (Constante) & 4.406 & .294 & & 14.993 & .000 & & \\
\hline & Género & -.045 & .099 & -.019 & -.448 & .654 & .966 & 1.035 \\
\hline & Universidad & -.307 & .067 & -.194 & -4.568 & .000 & .953 & 1.049 \\
\hline & Grupo étnico & -.028 & .015 & -.077 & -1.839 & .067 & .972 & 1.029 \\
\hline & Satisfacción con la vida & .408 & .042 & .405 & 9.661 & .000 & .978 & 1.022 \\
\hline \multirow[t]{6}{*}{3} & (Constante) & 3.566 & 391 & & 9.113 & .000 & & \\
\hline & Género & -.038 & .098 & -.016 & -.382 & .703 & .965 & 1.036 \\
\hline & Universidad & -.321 & .067 & -.203 & -4.820 & .000 & .949 & 1.054 \\
\hline & Grupo étnico & -.027 & .015 & -.074 & -1.765 & .078 & .971 & 1.030 \\
\hline & Satisfacción con la vida & .369 & .044 & .366 & 8.448 & .000 & .899 & 1.112 \\
\hline & Autoestima & .336 & .105 & .138 & 3.209 & .001 & .915 & 1.092 \\
\hline
\end{tabular}




\section{DISCUSIÓN}

Los miembros de los pueblos originarios en países latinoamericanos históricamente se han enfrentado a diferentes barreras que han impedido el acceso a la educación (Johnson, 2015). Bajo este contexto, es que la universidad intercultural surgió en México, en el año 2004, y a partir de dicha fecha, se han establecido 12 universidades en diferentes regiones del país donde habitan miembros de algún pueblo originario (Mateos y Dietz, 2016). Es importante señalar que contrarrestar la pobreza ha sido un objetivo común, tanto para la educación financiera (Loomis, 2018), como de la universidad intercultural en México (Tapia, 2016); por lo tanto, sería importante reflexionar acerca de cuáles elementos podrían ser integrados para favorecer a la educación financiera de los estudiantes interculturales dentro de un modelo relativamente nuevo, el cual se fundamenta en la inclusión, no solo de personas, sino también de ideas y metodologías.

La presente investigación, desde la parte descriptiva, refleja que los estudiantes interculturales mostraron niveles favorables de satisfacción con la vida y autoestima, a través de los resultados obtenidos luego de aplicar el SWLS (Diener et al., 1985) y el RES (Ronsenberg, 1965), respectivamente. Aquí, es de resaltarse que la evidencia empírica acerca de dichas variables dentro del contexto intercultural no es del todo vasta. Si bien es cierto que ambos cuestionarios han sido analizados mediante estudios de invarianza de medida -lo cual corresponde a la validez de constructo- mediante la comparación entre diferentes grupos culturales 0 nacionalidades, su interpretación en contextos interculturales todavía no es del todo concluyente.

Dentro de la presente investigación se planteó una pregunta de investigación: ¿Cómo influye la satisfacción con la vida y la autoestima sobre el optimismo financiero en estudiantes interculturales de México? Con el propósito de responder ésta cuestión, se formularon dos hipótesis, las cuales, a través del sustento teórico que involucra tanto a la psicología positiva como a la economía conductual, se postuló que ambas variables independientes tienen un efecto positivo sobre el optimismo financiero. Si bien el estudio de la educación financiera no es del todo vasto dentro del entorno intercultural, existen dos posturas que se complementan entre sí: la perspectiva individual y la cultural. Primeramente, según Blue (2019), la educación financiera hace referencia a las características de los individuos, es decir de las personas. Aquí, el optimismo financiero, visto como atributo personal, puede ser comprendido a través de otros aspectos relacionados con el optimismo en general (Dawson, 2017), donde es posible considerar a la satisfacción con la vida y la autoestima.

La primera es un juicio cognitivo acerca del nivel de satisfacción respecto a las expectativas personales (Diener et al., 1985), donde también se considera la parte económica (Veenhoven, 2015), aspecto que liga a la satisfacción con la vida con el optimismo financiero, el cual, al igual que la variable anterior, también involucra la expectativa del futuro, pero en este caso, respecto a lo económico (White et al., 2020). Esto es que ambas tienen un punto contacto en cuanto al bienestar económico; lo cual se puede constatar en que la satisfacción con la vida ha sido empleada como parte del bienestar subjetivo (Pavot y Diener, 2008). En cuanto a la autoestima, que es considerada como una autovaloración (Rosenberg, 1965) positiva de un individuo (APA, 2010); ésta no puede ser explicada a través de lo económico sino viceversa, ya que la necesidad de logro financiero puede ser asociada con baja autoestima (Ward et al., 2021). Es así que, desde la parte individual, tanto satisfacción de la vida como la autoestima explican al optimismo financiero, lo cual fue verificado empíricamente en este estudio.

Por otro lado, la conducta humana también obedece a una parte social (APA, 2010). En este tenor, desde la perspectiva cultural, que posee un lugar central en esta investigación, se resalta que la cultura interviene en la comprensión de las variables independientes ya mencionadas (Krys et al., 2021; Salzman, 2018), y en cómo éstas son percibidas por personas que forman parte de diferentes grupos sociales, como es el caso del entorno intercultural. Asimismo, la cultura juega un papel relevante para la comprensión del optimismo financiero (White et al., 2021). Por lo tanto, se torna relevante hacer comparaciones entre grupos culturales para poder identificar diferencias, y considerar otras variables explicativas integradas en la psicología positiva y la economía conductual dentro del contexto intercultural. En consecuencia, esto demanda mayores investigaciones en este tipo de escenarios en la región latinoamericana.

El presente estudio trató de aportar evidencia empírica que pueda, en un primer momento, adherirse al corpus de la psicología positiva y, además, que vincule a esta disciplina con la economía conductual. Finalmente, lo ya mencionado permite mejorar la comprensión de dichas variables dentro del entorno intercultural, en especial dentro del modelo de universidad intercultural de México, donde se está intentando crear un escenario a través del cual los miembros de diferentes grupos culturales puedan comunicarse (Dietz y Mateos, 2020), por medio de un diálogo entre las diferentes culturas (Johnson, 2015). Si bien es cierto que el empoderamiento de los miembros de los grupos étnicos a través del fomento de la autoestima se está convirtiendo en un quehacer relevante dentro de la universidad intercultural en este país (Dietz y Mateos, 2020; Martí y Dietz, 2014), por lo tanto, el tomar a la psicología positiva y a la economía conductual como ejes transversales dentro de los programas ofertados por estas universidades podría ser una estrategia eficaz para contrarrestar los problemas de marginación dentro de la población intercultural que asiste a dichas 
instituciones educativas. A través de una muestra conformada por estudiantes universitarios de tres Instituciones interculturales de Educación Superior, se encontró que variables como la satisfacción con la vida y la autoestima tienen un impacto positivo y significativo sobre el desarrollo del optimismo financiero, aspecto individual que se basa en las expectativas favorables acerca del futuro (Jacobsen et al., 2014), en especial ante situaciones financieras inciertas (Talwar et al., 2021).

Cabe señalar que, aunque fue posible obtener datos de tres escenarios distintos, como es el caso de Sonora, Sinaloa y Tabasco, al ser México un país con una gran diversidad étnica (INALI, 2018), tal como lo muestra la Tabla 1, solo fue posible obtener información de estudiantes pertenecientes a 13 grupos culturales. De este modo, la cantidad de la muestra y el tipo de muestreo llevado a cabo constituyen las principales limitaciones del estudio; no obstante, esto no fue impedimento para poner a prueba las hipótesis planteadas. En este sentido, se resalta que los hallazgos presentados fueron suficientes para soportar empíricamente tal postulado, donde fue posible obtener resultados favorables para la confiabilidad y la validez de los instrumentos de medición. También se resalta que el análisis VIF mostró resultados adecuados que muestran que la regresión no presentó posibles problemas de colinealidad.

Si bien la universidad intercultural pretende generar desarrollo socioeconómico de las comunidades de los pueblos originarios en México (Mateos y Dietz, 2016), el fomento de la autoestima y la satisfacción con la vida de sus estudiantes, al mismo tiempo que podría tener un impacto en positivo sobre el optimismo financiero variable relevante dentro de la educación financiera-, también podrían servir para desarrollar diferentes capacidades de los alumnos, tales como el emprendimiento (Dawson et al., 2017). Esto debido a que el desarrollo del ser humano se ha convertido en objeto de estudio tanto la economía conductual (Zabelina et al., 2018), como de la psicología positiva (Seligman y Pawelski, 2003). Aunque ambas disciplinas son diferentes, éstas convergen en dicho interés común. A través de éstas podría ser posible una mejor comprensión de la educación intercultural, donde la evidencia empírica no es vasta.

\section{CONCLUSIONES}

La presente investigación se centra en variables que pueden servir para explicar la aportación de la educación financiera dentro del modelo de la universidad intercultural en México. Sus conclusiones y aportación se presentan a continuación: 1) los cuestionarios empleados mostraron niveles favorables de validez y confiabilidad, esto a pesar que muchos de los participantes del estudio, como miembros de algún pueblo originario de México, no tienen al español como lengua principal; 2) los estudiantes interculturales mostraron niveles altos en la evaluación de la satisfacción con la vida, autoestima y optimismo financiero; 3) los hallazgos reflejaron que las variables del estudio tienen una relación significativa y positiva dentro de entornos interculturales; 4) el optimismo financiero fue influenciado por la satisfacción con la vida y la autoestima; 5) esta investigación propone que variables que pertenecen tanto la economía conductual como a la psicología positiva podrían apoyar de manera conjunta en la comprensión y fomento de la educación financiera en grupos que históricamente han tenido problemas de rezago y marginación, como lo son los pueblos originarios de Latinoamérica.

\section{AGRADECIMIENTOS}

Los autores agradecen al Programa de Fomento a la Investigación (PROFAPI) 2020 del Instituto Tecnológico de Sonora (ITSON), que apoyó el presente proyecto de investigación.

\section{REFERENCIAS}

Asociación Americana de Psicología., APA Diccionario conciso de psicología, $1^{a}$ ed., Manual Moderno, México (2010)

Blue, L.E., Financial literacy education with an aboriginal community: Identifying critical moments for enabling praxis, https://doi.org/10.3390/educsci9010012, Education Sciences, 9(1), 1-13 (2019).

Consejo Nacional de Evaluación de la Política de Desarrollo Social., Informe de evaluación de la política de desarrollo social 2020, $1^{\text {a }}$ ed., CONEVAL, Ciudad de México, México (2020)

Dawson, C., Financial optimism and entrepreneurial satisfaction, https://doi.org/10.1002/sej.1244, Strategic Entrepreneurship Journal, 11(2), 171-194 (2017).

Diener, E., Emmons, R.A., y otros dos autores, The satisfaction with life scale. https://doi.org/10.1207/s15327752jpa4901_13, Journal of Personality Assessment, 49(1), 71-75 (1985)

Dietz, G., y Mateos, L.S., Entre comunidad y universidad: Una etnografía colaborativa con jóvenes egresadas/os de una universidad intercultural mexicana, http://doi.org/10.11156/aibr.150205, Revista de Antropología lberoamericana, 15(2), 273-299 (2020)

Fonseca, D., de Melo, F., y de Melo, B.C., Behavioral economics and behavioral finance: a bibliometric analysis of the scientific fields, https://doi.org/10.1111/joes.12262, Journal of Economic Surveys, 33(1), 3-24 (2019) 
García, J., Rueda, y otros dos autores, Indigenous peoples, exclusion and precarious work: design of strategies to address poverty in indigenous and peasant populations in Ecuador through the SWOT-AHP methodology, https://doi.org/10.3390/ijerph18020570, International Journal of Environmental Research and Public Health, 18(2), 1-20 (2021)

Instituto Nacional de Lenguas Indígenas, Catálogo de las lenguas indígenas nacionales. Cuaderno informativo. $1^{\text {a }}$ ed., 2 3, Secretaría de Cultura, Ciudad de México, México (2018)

Instituto Nacional de los Pueblos Indígenas, 100 días de gobierno, 1르 ed., Instituto Nacional de los Pueblos Indígenas, Ciudad de México, México (2018)

Johnson, D., Formar ciudadanos interculturales en un mundo global: algunas notas desde los estudios curriculares, https://dx.doi.org/10.4067/S0719-26812015000200002, Diálogo Andino, (47), 7-14 (2015)

Krys, K., Park, J., y otros sesenta, Personal life satisfaction as a measure of societal happiness is an individualistic presumption: evidence from fifty countries, https://doi.org/10.1007/s10902-020-00311-y, Journal of Happiness Studies, 22, 2197-2214 (2021)

Levinson, J. D., y Peng, K., Valuing cultural differences in behavioral economics, The ICFAI Journal of Behavioral Finance, ISSN: 0972-9089, 4(1), 32-47 (2007)

Loomis, J.M., Rescaling and reframing poverty: financial coaching and the pedagogical spaces of financial inclusion in Boston, Massachusetts, https://doi.org/10.1016/j.geoforum.2018.06.014, Geoforum, 95, 143-152 (2018)

Martí, S., y Dietz, G., Empoderamiento y educación superior en contextos interculturales en México. 1aㅡ ed., 11-16, Bellaterra, Barcelona, España (2014)

Martínez, P., Ovares, Y., y Garrido, N., Educación intercultural en el proceso de humanización de estudiantes universitarios: influencias en el rendimiento académico, Interciencia, 45(4), 201-208 (2020)

Mateos, L.S., y Dietz, G., Universidades interculturales en México. Balance crítico de la primera década, ISSN: 14056666, Revista Mexicana de Investigación Educativa, 21(70), 683-690 (2016)

Miranda, R.G., Pobreza, desigualdad y vulnerabilidad en América Latina (2000-2020), Americanía: Revista de Estudios Latinoamericanos, ISSN: 2174-0178, (11), 56-90 (2019).

Núñez, M.A., Banegas, R.A., y otros dos autores, El lado positivo del emprendedor universitario. Autoestima, satisfacción con la vida y optimismo en estudiantes de México y Bolivia, http://dx.doi.org/10.4067/S071850062020000400021, Formación Universitaria, 13(4), 21-30 (2020)

Pavot, W., y Diener, E., The satisfaction with life scale and the emerging construct of life satisfaction, http://dx.doi.org/10.1080/17439760701756946, The Journal of Positive Psychology, 3(2), 137-152 (2008)

Rosenberg, M., Society and the adolescent self-image, $1^{\underline{a}}$ ed., Princeton University Press, Princeton, NJ (1965)

Salzman, M.B, Culture and self-Esteem, in a psychology of culture, International and Cultural Psychology, $1^{1 \mathfrak{a}}$ ed., Springer, Cham, 43-54, Honolulu, USA (2018)

Seligman, M.E.P., y Pawelski, J., Positive psychology: FAQs, Psychological Inquiry, ISSN: 1532-7965, 14(2), 159-163 (2003)

Talwar, M., Talwar, S., y otros tres autores, Has financial attitude impacted the trading activity of retails investors during the COVID -19 pandemic? https://doi.org/10.1016/j.jretconser.2020.102341, Journal of Retailing and Consumer Services, 58, 1-11 (2021)

Tapia, L.A., El Subsistema de Universidades Interculturales en México. Entre la política social y la política educativa, Revista Latinoamericana de Estudios Educativos, ISSN: 0185-1284, 46(1), 7-50 (2016)

Veenhoven, R., Overall satisfaction with life. Subjective approaches, In Global handbook of quality of life. Exploration of well-being of nations and communities, by W. Glatzer, L. Camfield, L., V. Moller, y M. Rojas, 207-238, 1를. ed., Springer, Dordrecht, Netherlands (2015)

Villalta, M.A., Educación intercultural en Latinoamérica: Análisis de las investigaciones de campo en la región, Psicoperspectivas. Individuo y Sociedad, ISSN: 0717-7798, 15(1), 118-131 (2016)

Ward, D., Park, L.E., y otros tres autores, Can't buy me love (or friendship): social consequences of financially contingent self-worth, https://doi.org/10.1177/0146167220910872, Personality and Social Psychology Bulletin, 46(12), 1-17 (2020)

White, K., Watkins, K., y otros tres autores, How financial socialization messages relate to financial management, optimism and stress: variations by race, https://doi.org/10.1007/s10834-020-09704-w, Journal of Family and Economic Issues, 42, 237-250 (2021)

Zabelina, E., Tsiring, D., y Chestyunina, Y., Personal helplessness and self-reliance as the predictors of small business development in Russia: pilot research results, https://doi.org/10.1007/s11365-018-0502-y, International Entrepreneurship and Management Journal, 14, 279-293 (2018) 
